\title{
Bethe-Salpeter Kernel and Short Distance Expansion in the Massive Gross-Neveu Model
}

\author{
D. Iagolnitzer ${ }^{1}$ and J. Magnen ${ }^{2}$ \\ 1 Service de Physique Théorique de Saclay, ${ }^{\star}$ F-91191 Gif-sur-Yvette Cedex, France \\ 2 Centre de Physique Théorique, ${ }^{\star \star}$ Ecole Polytechnique, F-91128 Palaiseau Cedex, France
}

\begin{abstract}
The Bethe-Salpeter kernel is defined (non-perturbatively) for the weakly coupled massive Gross-Neveu model. Its large momentum properties are established. They are used to justify "subtracted" Bethe-Salpeter equations initially proposed (for $\varphi_{4}^{4}$ ) by K. Symanzik, and in turn to give non-perturbative proofs of the Wilson short distance expansion at first order and of 2-particle asymptotic completeness and related results.
\end{abstract}

\section{Table of Contents}

1. Introduction . . . . . . . . . . . . . . . . . . . . . 567

1.1. Background . . . . . . . . . . . . . . . . . . . . 567

1.2. Description of Contents and Results . . . . . . . . . . . . 569

2. Symanzik's Subtracted Bethe-Salpeter Equations (Formal Derivation) . 571

3. The Bethe-Salpeter Kernel. . . . . . . . . . . . . . . . . 573

4. Large Momentum Properties of Bethe-Salpeter Kernel. . . . . . . 575

5. Applications . . . . . . . . . . . . . . . . . . . . . 577

5.1. Wilson Short-Distance Expansion . . . . . . . . . . . . 577

5.2. 2-Particle Asymptotic Completeness and Related Results . . . . 579

6. Discussion . . . . . . . . . . . . . . . . . . . . . . 582

References . . . . . . . . . . . . . . . . . . . . . 584

\section{Introduction}

1.1. Background. Recent non-perturbative works, which apply in particular to the weakly coupled massive Gross-Neveu model [GN, MW] in dimension 2, constructed in [FMRS, GK], have been made in two directions

(i) 2-particle asymptotic completeness (AC) and related results on scattering

* Laboratoire de l'Institut de Recherche Fondamentale du Commissariat à l'Energie Atomique

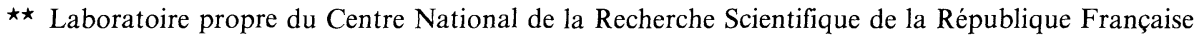


have been established [IM1, BI] through the use of a $2 \rightarrow 2$ kernel $\widehat{G}$ which does not have a simple perturbative content but is well defined [IM1] from "particle analysis" made at low energies only and is linked to the (true) connected, amputated 4-point function $F$ by a regularized Bethe-Salpeter equation with (fixed) ultraviolet cut off:

$$
F=\hat{G}+\widehat{G} o_{M} F,
$$

where $o_{M}$ denotes Feyman-type convolution with 2-point functions modified by (fixed) cut-off factors on internal lines. (The cut-off is linked to the parameter $M$ that defines the width of the lowest momentum slice in phase-space analysis: see below. The dependence of $\hat{G}$ on the choice of $M$ is left implicit.)

Recourse to the kernel $\hat{G}$ is a simple way, following the axiomatic ideas of [B1, BL] of avoiding ultraviolet problems, expected to prevent direct use of the Bethe-Salpeter equation in non-super-renormalizable theories.

(ii) Large momentum properties of Green functions have been established in [IM2]. Let $k=p_{1}+p_{2}, z=\left(p_{1}-p_{2}\right) / 2, z^{\prime}=\left(p_{3}-p_{4}\right) / 2$. For $(N+2)$-point functions $(N>2), z^{\prime}$ will more generally denote a set of $N-1$ relative energy-momenta among $p_{3}, \ldots, p_{N+2}$. We are interested below in results as $z$ (which is the dual variable of $x_{1}-x_{2}$ ) tends to infinity in euclidean space, $k, z^{\prime}$ fixed. It is first shown in [IM2] that $F_{4}$ or $F_{N+2}$ behaves like $1 /(\ln |z|)^{1-\sigma}$ as $z \rightarrow \infty$. $(\sigma$ depends on the channel determined by $(1,2)$ : fields $\psi$ or $\bar{\psi}$, colour and spin indices.) Results of [IM2] then include a non-perturbative proof of Wilson-Zimmerman short-distance expansion $[\mathrm{W}, \mathrm{Z}, \mathrm{S}]$. At first order, the latter can be written for the set of functions $F_{N+2}, N \geqq 2$, in the form:

$$
F_{N+2}\left(k, z, z^{\prime}\right)=\frac{\omega(0, z)}{\omega(k, z)} F_{4}(0, z, 0) \Lambda_{N}\left(k, z^{\prime}\right)+R_{N+2}\left(k, z, z^{\prime}\right)
$$

where $\omega(k, z)$ is the product $S\left(p_{1}\right) S\left(p_{2}\right)$ of 2-point functions $\left(p_{1}=k / 2+z\right.$, $\left.p_{2}=k / 2-z\right), \Lambda_{N}, R_{N+2}$ are well defined (finite) functions, and $R_{N+2}$ is shown in [IM2] to satisfy (for the GN model) the bound:

$$
\left|R_{N+2}\left(k, z, z^{\prime}\right)\right|<\frac{C_{N}\left(k, z^{\prime}\right)}{1+|z|}\left[\operatorname{Inf}\left(\lambda_{\text {ren }}, \frac{1}{\ln (1+|z|)}\right)\right]^{1-\sigma} .
$$

The euclidean version of this result ( $k, z^{\prime}$ euclidean) is established in [IM2] via a method that is in some sense an analogue of the perturbative method using composite operators and does not use Bethe-Salpeter (BS) or BS-type kernels. For field theory versions of the Wilson expansion, Minkowskian values of $k, z^{\prime}$ have to be considered. Results in this direction are achieved in [IM2], for values of $k$ up to $s=16 \mu^{2}-\eta(\eta>0)$ in Minkowski space by an adaptation of this method using the BS-type kernel $\hat{G} ; \mu>0$ is here the basic physical mass of the theory.

A different way of understanding Wilson expansion at first order, e.g. at $k, z^{\prime}$ euclidean, has been proposed by K. Symanzik [S], and recently reconsidered and developed in [BD]. It applies more particularly to "à la $\varphi_{4}^{4}$ " theories, that will include the GN model, whose renormalization parts are 2 and 4-point functions, and is based on the use of the "true" BS kernel G (or of the "renormalized" BS kernel $G_{\text {ren }}$ : see note at the end of Sect. 1.2). More precisely, "subtracted" 
Bethe-Salpeter equations are proposed in [S]. They provide formal expressions of $\Lambda_{N}, R_{N+2}$ in terms of $G$, but only through differences $G-\stackrel{\circ}{G}, \stackrel{\circ}{G}-\stackrel{\circ}{G}_{0}$, where $\stackrel{\circ}{G}\left(k, z, z^{\prime}\right)=G\left(0, z, z^{\prime}\right), \stackrel{\circ}{G}_{0}\left(k, z, z^{\prime}\right)=G(0, z, 0)$. E.g. at $k=0, N=2, R_{4}$ is (formally) an infinite sum of Feynman-type multiple convolution products of $\stackrel{\circ}{G}-\stackrel{\circ}{G}_{0}$ (see formula (15) in Sect. 2). The following idea is then suggested: these differences should have better decrease properties than $G$ itself as $z \rightarrow \infty$, that should allow one to give sense to these formulae and to derive the decrease of $R_{N+2}$.

On the other hand, methods for the derivation of 2-particle AC and related results also based on the use of the kernel $G$ (or $G_{\text {ren }}$ ), rather than $\hat{G}$, have been proposed in [I1, BD]. It is in particular remarked in [BD] that one of Symanzik's equations, which expresses $F$ (at $N+2=4$ ) in terms of $\stackrel{\circ}{\equiv} F\left(0, z, z^{\prime}\right)$ and of $G-\stackrel{\circ}{G}$ might allow one to define the analytic continuation of $F$ to the 2-particle region in Minkowski space needed for this derivation.

1.2. Description of Contents and Results. These proposals are implemented in this work in a precise way for the GN model. A comparison of the methods of this paper with those of [IM1, IM2] and a related discussion will be given at the end (Sect. 6).

Symanzik's equations are rederived (formally) in Sect. 2.

In Sect. 3, we first briefly recall the non-perturbative construction of the GN model, following [FMRS, IM1]. This construction is made through "phase-space analysis" and involves the introduction of "momentum slices," the $i^{\text {th }}$ momentum slice corresponding to momenta of the order of $M^{i}$. The $2 \rightarrow 2$ BS kernel $G$ is then defined as the sum, convergent at small coupling, of phase-space diagrams that are 2-particle irreducible (2-p.i.), in the $2 \rightarrow 2$ channel considered with respect to propagator lines of all momentum slices. The 2-particle irreducibility of contributing diagrams will be used in two different ways. On the one hand, it yields, as in the case of $\hat{G}$, analyticity of $G$ in a strip around euclidean space going up to $s=16 \mu^{2}-\eta(\eta>0)$ in Minkowski space. On the other hand, large momentum properties of $G$ that are simpler than those expected (and proved in [IM2]) for $F$ follow from 2-particle irreducibility with respect to different slices: a connected subdiagram obtained in slices $\geqq j$ from a cut at a given slice $j$ and including the external lines 1,2 (but not 3,4 ) cannot have two outgoing lines only below $j$ (unless these lines are the external lines 3,4$)$. In view of the fact that the only renormalization parts in the GN model are 2 and 4-point functions, differences $G-G, G-G_{0}$ are then shown to have indded a better decrease than $F$ or $G$ as $z \rightarrow \infty$.

More precisely the following bounds on $G, G-\stackrel{\circ}{G}, G-G_{0}$ as also on $2 \rightarrow N$ 2-p.i. kernels $L_{N+2}$ (defined in a way similar to $G$ ) are established in Sect. 4 for $k, z^{\prime}$ euclidean. (The extension to non-euclidean values is presented in Sect. 5.2.) Although results to be established in the applications, e.g. on $R_{N+2}$, apply at $k, z^{\prime}$ fixed, their proof requires results on the differences $G-\stackrel{\circ}{G}, G-G_{0}$ (involved in multiple convolutions) as both $z$ and $z^{\prime}$ tend to infinity. On the other hand, results on $L_{N+2}, N>2$, are needed (and thus stated below) only at $k, z^{\prime}$ fixed.

Proposition 1. $\exists \lambda_{0}>0$ such that:

1) $\left|G\left(k, z, z^{\prime}\right)\right| \leqq f(k) \operatorname{Inf}\left\{\lambda_{\text {ren }}, \frac{1}{\ln (1+|z|)}\right\} \quad$ if $|z| \geqq 3\left|z^{\prime}\right|$, 


$$
\left|G\left(k, z, z^{\prime}\right)\right| \leqq f(k) \operatorname{Inf}\left\{\lambda_{\text {ren }}, \frac{1+\ln (1+\ln (1+|z|))}{\ln (1+|z|)}\right\} \text { if }|z| \geqq\left|z^{\prime}\right|
$$

$$
\left(|z| \leqq 3\left|z^{\prime}\right|\right)
$$

for $\lambda_{\text {ren }}<\lambda_{0}$.

2) $\forall \varepsilon>0, \lambda_{\text {ren }}<\lambda_{0} \varepsilon$

$\begin{array}{ll}\text { a) } & \mid\left(G-\stackrel{\circ}{G}\left(k, z, z^{\prime}\right) \mid<f(k) \frac{\lambda_{\text {ren }}^{2}}{(1+|z|)^{1-\varepsilon}} \quad \text { if }|z| \geqq\left|z^{\prime}\right|,\right. \\ \left|\left(G-G_{0}\right)\left(k, z, z^{\prime}\right)\right|<f(k) \lambda_{\text {ren }}^{2}\left(\frac{1+\left|z^{\prime}\right|}{(1+|z|)}\right)^{1-\varepsilon} \quad \text { if }|z| \geqq\left|z^{\prime}\right|,\end{array}$

b) $\left|L_{N+2}\left(k, z, z^{\prime}\right)\right|<f_{N}\left(k, z^{\prime}\right) \lambda_{\text {ren }}^{2} \frac{1}{(1+|z|)^{1-\varepsilon}}$.

Proposition 1 will allow one in turn to give sense to Symanzik's equations and to prove in Sect. 5

Proposition 1.1. $\forall \varepsilon>0, \exists \lambda_{0}^{\prime}>0$ such that Eq. (1) holds with

$$
\left|R_{N+2}\left(k, z, z^{\prime}\right)\right|<C_{N}\left(k, z^{\prime}\right) \frac{\lambda_{\text {ren }}^{2}}{(1+|z|)^{1-2 \varepsilon}}
$$

for $\lambda_{\text {ren }}<\lambda_{0}^{\prime} \varepsilon$.

Proposition 1.2. For any given $\eta>0, \exists \lambda_{0}^{\prime \prime}>0$ such that 2-particle asymptotic completeness holds in the region $(2 \mu)^{2}<s<(4 \mu)^{2}-\eta$ for $\lambda_{\text {ren }}<\lambda_{0}^{\prime \prime}$.

Related results on the analytic structure of $F$ are also obtained.

Bounds better than (3) $\left(3^{\prime}\right)\left(3^{\prime \prime}\right)$, in which $\varepsilon$ is removed can also be established and are given in Prop. 2 below. They yield in turn an improvement of the bounds on $R_{N+2}$ (Prop. 2.1). (There is no appreciable improvement of Prop. 1.2.) However, the proof of Prop. 2 requires recourse to methods of [IM2] for treating the Wilson expansion up to second order. The advantage of using the BS kernel to prove the Wilson expansion is then lost and the proof is therefore omitted.

Proposition 2. $\exists \lambda_{0}>0$ such that $\forall \lambda_{\text {ren }}<\lambda_{0},|z| \geqq 3\left|z^{\prime}\right|$,

$$
\begin{gathered}
\left|\left(G-G^{\circ}\right)\left(k, z, z^{\prime}\right)\right|<\frac{g(k)}{1+|z|} \operatorname{Inf}\left\{\lambda_{\text {ren }}^{2}, \frac{1}{\left(1+\ln (1+|z|)^{2}\right.}\right\}, \\
\left|\left(G-G_{0}\right)\left(k, z, z^{\prime}\right)\right|<g(k) \frac{1+\left|z^{\prime}\right|}{1+|z|} \operatorname{Inf}\left\{\lambda_{\text {ren }}^{2}, \frac{1}{\left(1+\ln (1+|z|)^{2}\right.}\right\}, \\
\left|L_{N+2}\left(k, z, z^{\prime}\right)\right|<g_{N}\left(k, z^{\prime}\right) \frac{1}{1+|z|} \operatorname{Inf}\left\{\lambda_{\text {ren }}^{2}, \frac{1}{(1+\ln (1+|z|))^{2}}\right\} .
\end{gathered}
$$

Part 1 of Prop. 1 and Prop. 2 yield in turn the following improvement of Prop. 1.1:

Proposition 2.1. $\exists \lambda_{0}>0$ such that $\forall \lambda_{\text {ren }}<\lambda_{0}$, Eqs. $(1)\left(1^{\prime}\right)$ hold.

The extension of results to values of $k$ up to $s=16 \mu^{2}-\eta$ in Minkowski space and the (related) proof of 2-particle asymptotic completeness and related results 
are given in Sect. 5.2. In both cases, the analysis of the neighborhood of the 2-particle threshold (where, as is well known, special problems occur in dimension 2 ) is made by introducing the same kernel $U$ as in [I2, BI], which in the present approach is defined directly in terms of $G$ (rather than $\hat{G}$ ) through the differences $G-\stackrel{\circ}{G}, \stackrel{\circ}{G}-\stackrel{\circ}{G}_{0}$.

Note. $G^{(\rho)}$ and $G_{\text {ren }}^{(\rho)}$ are, perturbatively, sums of non-renormalized 2-p.i. Feynman graphs with bare coupling and of renormalized 2-p.i. graphs with renormalized coupling respectively, in the theory with ultraviolet cut-off (depending on the parameter $\rho$ ). The kernel $G_{\mathrm{ren}}^{(\rho)}$ may à priori seem more likely in à la $\varphi_{4}^{4}$ theories to have a well defined $\rho \rightarrow \infty$ limit $G_{\text {ren }}$ and is for that reason the kernel considered in [I1, BD]. However, both $G$ and $G_{\text {ren }}$ will be well defined in the $\rho \rightarrow \infty$ limit in the GN model, due to asymptotic freedom.

As explained in [I1] and in the remark at the end of Sect. 3, $G^{(\rho)}$ and $G_{\text {ren }}^{(\rho)}$, or their $\rho \rightarrow \infty$ limits, differ only by a constant in theories whose renormalization part are only 2 and 4-point functions, so that either one can be used for the GN model in Symanzik's equations.

\section{Symanzik's Subtracted Bethe-Salpeter Equations (Formal Derivation)}

All equations below can be considered either in the theory with ultraviolet cut-off, depending on $\rho$, or in the $\rho \rightarrow \infty$ limit; $\rho$ will be left implicit. Feynman-type convolutions $A \circ B$ with 2-point functions will be denoted $A \omega B$ :

$$
(A \omega B)\left(k, z, z^{\prime}\right)=\int A(k, z, \zeta) \omega(k, \zeta) B\left(k, \zeta, z^{\prime}\right) d \zeta,
$$

where $\omega(k, z)$ is defined in Sect. 1.

$A \stackrel{\omega}{B}$ is defined similarly with $\omega(k, \zeta)$ replaced by $\omega(0, \zeta)$ in the integration measure. More generally, terms $A \omega B \omega C \ldots$ with $\omega$ possibly replaced by $\stackrel{\omega}{\text { are }}$ multiple convolution integrals over successive variables $\zeta_{1}, \zeta_{2}, \ldots$. Functions $\omega$ or $\stackrel{\circ}{\omega}$, or $\omega-\stackrel{\circ}{\omega}, \ldots$ on the left are not integrated. E.g.,

$$
\omega F\left(k, z, z^{\prime}\right) \equiv \omega(k, z) F\left(k, z, z^{\prime}\right) .
$$

But

$$
\begin{aligned}
(1 \omega F)\left(k, z, z^{\prime}\right) \equiv(1 \omega F)\left(k, z^{\prime}\right) & \equiv \int 1(k, z, \zeta) \omega(k, \zeta) F\left(k, \zeta, z^{\prime}\right) d \zeta \\
& \equiv \int \omega(k, z) F\left(k, z, z^{\prime}\right) d z .
\end{aligned}
$$

By convention,

$$
\stackrel{ }{A}\left(k, z, z^{\prime}\right) \equiv \AA \text { A }\left(z, z^{\prime}\right) \equiv A\left(0, z, z^{\prime}\right) .
$$

We define

$$
\begin{gathered}
B=G-G_{0}, \\
V=(\omega-\stackrel{\circ}{)} F+\stackrel{\circ}{\omega}(G-\stackrel{\circ}{G})+\stackrel{\circ}{\omega}(G-\stackrel{\circ}{G}) \omega F,
\end{gathered}
$$

with, by convention:

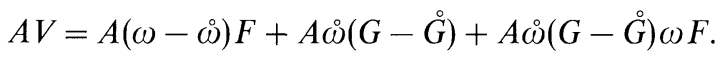


Starting from the BS equations:

$$
\begin{gathered}
F_{4}=G+G \omega F_{4}=G+F_{4} \omega G, \\
F_{N+2}=L_{N+2}+F_{4} \omega L_{N+2},
\end{gathered}
$$

we derive below:

\section{Lemma 1.}

$$
F_{N+2}\left(k, z, z^{\prime}\right)=\frac{\omega(0, z)}{\omega(k, z)} F_{4}(0, z, 0) \Lambda_{N}\left(k, z^{\prime}\right)+R_{N+2}\left(k, z, z^{\prime}\right)
$$

with

$$
\begin{gathered}
\stackrel{\circ}{R}_{4}\left(z, z^{\prime}\right)=[1-\stackrel{\circ}{B}]^{-1}\left(z, z^{\prime}\right)-1 \equiv \stackrel{\circ}{B}+\stackrel{\circ}{\circ} \stackrel{\circ}{B}+\stackrel{\circ}{\circ} \stackrel{\circ}{B} \stackrel{\circ}{\circ} \stackrel{\circ}{B}+\cdots, \\
\omega R_{4}=\stackrel{\circ}{\omega} R_{4}+V+\stackrel{\circ}{\circ} R_{4} V, \\
R_{N+2}=L_{N+2}+R_{4} \omega L_{N+2}, \\
\Lambda_{N}=\delta_{2, N}+1 \omega R_{N+2} .
\end{gathered}
$$

The following result will be derived in the course of the proof of Lemma 1 $(N=2$ is left implicit):

$$
\omega F=\stackrel{\circ}{\circ}+\stackrel{\circ}{F} V+V .
$$

It will be useful in Sect. 5.2 to rewrite this equation (by subtracting $(\omega-\stackrel{\circ}{)} F$ from both sides, dividing by $\stackrel{\circ}{\circ}$ and regrouping terms involving $F$ ) in the form

\section{Lemma 2.}

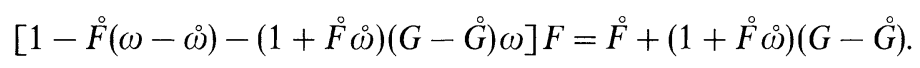

On the other hand, we note that at $\rho$ finite, $\Lambda_{N}$ is also equal (as follows from (14) and (18): see e.g. [IM2]) to:

$$
\Lambda_{N}\left(k, z^{\prime}\right)=\frac{\delta_{2, N}+\left(1 \omega F_{N+2}\right)\left(k, z^{\prime}\right)}{1+\left(1 \omega F_{4}\right)(0,0)} .
$$

Quantities $1 \omega F_{N+2}$ and $1 \omega F_{4}$ will become infinite in the $\rho \rightarrow \infty$ limit, $\Lambda_{N}$ remaining, however, finite in that limit.

\section{Proof of Lemma 1.}

a) $N=2, \quad k=0$.

We replace $\stackrel{\circ}{G}$ by $\stackrel{\circ}{B}+\stackrel{\circ}{G}_{0}$ in the expansion $\stackrel{\circ}{F}=\stackrel{\circ}{G}+\stackrel{\circ}{\omega} \stackrel{\circ}{G}+\stackrel{\circ}{G}^{\circ} \stackrel{\circ}{G} \omega^{\circ} \stackrel{\circ}{G}+\ldots$ of $\stackrel{\circ}{F}$ in terms of $\dot{G}^{\circ}$. Terms without factors $\dot{G}_{0}$ yield $\stackrel{R}{R}$ as defined in (15). Other terms are regrouped according to the last term $\dot{G}_{0}$ on the right. Factors $G_{0}$ and $\stackrel{\circ}{B}$ on

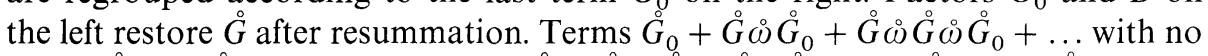

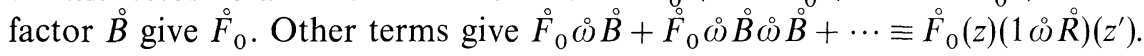

b) Proof of (19) $(N=2)$.

We start e.g. from the expansion $\omega F=\omega G+\omega G \omega G+\cdots$ and put $r=\omega G-$ $\omega \stackrel{\circ}{G}$, so that $\omega G=\omega \stackrel{\circ}{G}+r$. Contribution to $\omega F$ with no factor $r$ give $\dot{\omega} F$. Contributions with at least one factor $r$ are regrouped according to the first factor 
$r$ on the left. Resummation on the right of $\stackrel{\circ}{G}$ and $r$ restores $\omega G$. Using when needed the BS equation, this yields:

$$
\omega F=\stackrel{\circ}{\circ}+r+r \omega F+\stackrel{\circ}{\circ} r+\omega \stackrel{\circ}{F} r \omega F
$$

from which (19) follows by writing $r$ in the form $(\omega-\stackrel{\circ}{\omega} G+\stackrel{\circ}{\omega}(G-\stackrel{\circ}{G})$ and using again the BS equation (in the form $F=G+G \omega F$ ).

c) $N=2, \quad k \neq 0$.

Equation (19), together with Eq. (14) at $k=0$ and the definition (16) of $R$ at $k \neq 0$, yields:

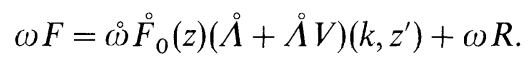

On the other hand, using $\stackrel{\Lambda}{=}=1+1 \stackrel{\circ}{R}$ and again the definition (16),

$$
\grave{\Lambda}+\stackrel{\Lambda}{V}=1+1 \omega R(\equiv \Lambda) \text {. }
$$

d) $N>2$.

Equation (14) at $N>2$ follows from (13), after replacing $F_{4}$ by its expression (14), taking into account the definition (17) of $R_{N+2}$ and using the equality:

$$
\Lambda_{2} \omega L_{N+2}=\Lambda_{N} \quad(N>2) \text {. }
$$

Equation (24) can be checked from the definition $\Lambda_{2}=1+1 \omega R_{4}$ which yields $\Lambda_{2} \omega L_{N+2}=1 \omega L_{N+2}+\left(1 \omega R_{4}\right) \omega L_{N+2}=1 \omega\left(L_{n+2}+R_{4} \omega L_{N+2}\right)=1 \omega R_{N+2}$.

\section{The Bethe-Salpeter Kernel $G$}

We first recall the phase space expansion of Green functions following [IM1]. A momentum cut-off is introduced in the theory by replacing the bare propagator $\widetilde{C}(p)$ (in momentum space) by $\widetilde{C}_{\rho}(p)=\widetilde{C}(p) \exp \left[-M^{-2 \rho}\left(p^{2}+m_{\rho}^{2}\right)\right] . \widetilde{C}_{\rho}$ is itself decomposed as $\sum_{i=1}^{\rho} \widetilde{C}^{(i)}(p)$ with e.g.:

$$
\begin{aligned}
\tilde{C}^{(1)}(p) & =\tilde{C}(p) \exp \left[-M^{-2}\left(p^{2}+m_{\rho}^{2}\right)\right] \\
\tilde{C}^{(i)}(p) & =\widetilde{C}(p)\left\{\exp \left[-M^{-2 i}\left(p^{2}+m_{\rho}^{2}\right)\right]-\exp \left[-M^{-2(i-1)}\left(p^{2}+m_{\rho}^{2}\right)\right]\right\}
\end{aligned}
$$

for $i>1$. A corresponding decomposition of $C(x)$ follows in euclidean space-time. Euclidean phase-space expansions of e.g. connected Green functions are obtained from (space-time) cluster expansions in each momentum slice $i=1, \ldots, \rho$ with respect to scaled lattices of size $M^{-i}$ and Mayer procedures. They express Green functions as sums of "phase-space diagrams" including propagator and Mayer lines (joining squares of the lattice) in each slice $i$, as also lines joining different slices (and linking together propagators attached to the same interaction vertex). The expansion is shown to be convergent for values of the bare coupling $\lambda$ of the form $|\lambda|<\operatorname{cst} M^{-\rho+\varepsilon / 2}$ as $\rho \rightarrow \infty$. This is not sufficient to get a non-trivial theory in the $\rho \rightarrow \infty$ limit. The renormalization procedure (= rearrangement of terms of the expansion, starting from slice $\rho$ and up to slice 1 , with redefinition of an effective coupling $\lambda_{i}$ for each momentum slice $i ; \lambda_{p} \equiv \lambda$ is the bare coupling) leads to a new expansion, shown to be convergent (and analytic in $\lambda$ ) in a larger circle that contains 
for each $\rho$ the points

$$
\lambda_{\rho}=\left[-\beta_{2}(\ln M) \rho+\beta_{3} \ln \rho+D\right]^{-1}, \quad\left(\beta_{2}<0\right)
$$

for $D$ sufficiently large (independent of $\rho$ ). The expansion is uniformly convergent, Green functions have well defined $\rho \rightarrow \infty$ limits and $\lambda_{1}$ is $\neq 0$ in that limit $\left(\lambda_{1} \approx 1 / D\right) ; \lambda_{\text {ren }}$, equal to $\lambda_{1}$ at first order in $\lambda_{1}$, is thus itself $\neq 0$ : non-triviality.

Although usual cluster expansions are sufficient for the above analysis, we shall here start for our purposes from "4th-order" cluster expansions [IM3, 2] in all slices: this gives somewhat more complicated expansions but convergence properties are still established in a similar way. It is more precisely convenient to first put aside bare propagators $C\left(x_{k}-y_{k}\right)$ linked to external points ("semi-amputation"). Corresponding phase-space expansions follow for connected, semi-amputated functions. The expansions of the connected amputated Green functions are obtained by restricting the sums to graphs that are $1-$ p.i. in all $1 \rightarrow N-1$ channels.

The BS kernel $G^{(\rho)}$ is defined as the contribution to the 4-point function $F^{(\rho)}$ composed, before renormalization. of graphs that are "totally" 2-p.i. in the $2 \rightarrow 2$ channel considered. They are in particular 1 -p.i. in $1 \rightarrow 3$ channels. As above, irreducibility is understood with respect to propagator lines of all momentum slices. (A 2-p.i. graph in the channel $(1,2),(3,4)$ cannot to separated into two parts, with external lines 1,2 and 3,4 on each side, by cutting 2 propagator lines or less.) As previously, this expansion is first shown to be convergent for values of the bare coupling $\lambda$ that tend to zero like $M^{-(\rho+\varepsilon) / 2}$ as $\rho \rightarrow \infty$; the BS equation (for each given $\rho$ ) is established by graphical inspection for these values of $\lambda$. Results below will allow extensions to the value (25) of $\lambda_{\rho}$ and in turn to the $\rho \rightarrow \infty$ limit.

The renormalization procedure, along the same lines as above, is applied to $G^{(\rho)}$. It gives the following results:

(i) for all diagrams that are not reduced to a single vertex, the effective coupling constants in each slice $i$ are the same $\lambda_{i}$ as before: this is due to the fact, analogous to that mentioned in the remark at the end of this section in the perturbative framework, that 2-particle irreducibility properties are not modified by replacing some vertices by 4-point subgraphs.

(ii) the situation is different for the trivial diagram with a single vertex. In this case, the flow of the coupling constant is modified: the effective coupling in each slice $i$ is given by

$$
\lambda_{i-1}^{\prime}=\lambda_{i}^{\prime}+\sum[2-\text { p.i. graphs }]
$$

where [ ] means the value at $k=z=z^{\prime}=0$ and where the graphs involved in $\left[\right.$ ] have effective coupling $\lambda_{j}$ in all slices $j \geqq i$.

Since there is a ratio $\gamma$ of 2-p.i. graphs of the form all graphs of this form Eq. (26) gives:

$$
\lambda_{i-1}^{\prime}=\lambda_{i}^{\prime}+\gamma\left(\beta_{2} \ln M\right) \lambda_{i}^{2}+\operatorname{cst} \lambda_{i}^{3}+\mathcal{O}\left(\lambda_{i}^{4}\right),
$$

which finally yields

$$
\lambda_{\text {ren }}^{\prime}=\gamma \lambda_{\text {ren }}+\mathcal{O}\left(\lambda_{\text {ren }}^{\prime 3}\right)
$$


The usual methods then show that the contribution $G^{\prime(\rho)}$ of the graphs of (i) to $G^{(\rho)}$ is still well defined for $\lambda_{\rho}$ of the form (25) and has a well defined limit $G^{\prime}$ as $\rho \rightarrow \infty$. $G$ is thus equal to

$$
G=\lambda_{\text {ren }}^{\prime}+G^{\prime}
$$

Remark. $G^{\prime}$ is equal from the perturbative viewpoint to the sum of renormalized 2-p.i. graphs not reduced to a single vertex. (This can be seen by completing the "useful" renormalization made along the lines of [FMRS] by "useless" ones.) The relation between $G_{\text {ren }}$ (see Sect. 1) and $G$ is thus:

$$
G_{\text {ren }}=G^{\prime}+\lambda_{\text {ren }}=G+(1-\gamma) \lambda_{\text {ren }}+\mathcal{O}\left(\lambda_{\text {ren }}^{3}\right) .
$$

The equality

$$
G_{\rho}=\left(G_{\mathrm{ren}}\right)_{\rho}+C_{\rho}
$$

where $C_{\rho}$ is a constant can be seen as follows from a perturbative viewpoint in "a la $\varphi_{4}^{4}$ " theories whose renormalization parts are at most 4-point functions such as the GN model (see a more compact argument in [I1]). The 2-particle irreducibility of a graph in the channel $(1,2),(3,4)$ is not modified if a vertex is replaced by any 4-point subgraph (or conversely), except for the trivial graph with a single vertex. The formal algebraic argument that shows that the sum of non-renormalized graphs with bare coupling is equal to the sum of renormalized graphs with renormalized coupling then yields (31). $C_{\rho}$ is perturbatively, e.g. the sum of non-renormalized 2-p.i. graphs with bare coupling, at $k=z=z^{\prime}=0$.

\section{Large Momentum Properties of Bethe-Salpeter Kernels}

The analysis of large momentum and short-distance properties of $G$ (at $\rho$ infinite) is analogous to that carried out for $F$ in [IM2], but important simplifications occur, at least in some situations, due to the 2-particle irreducibility of diagrams contributing to $G$.

We restrict here our attention to situations in which $k=p_{1}+p_{2}$ is fixed, while $z$ or $z^{\prime}$ or both will tend to infinity in euclidean space. External lines 1, 2, respectively 3,4 will belong by convention to slices $i(z), i\left(z^{\prime}\right)$ respectively. The new important facts, in comparison with the analysis of [IM2] are:

Remark 1. Let e.g. $i(z) \geqq i\left(z^{\prime}\right)$. Most contributing diagrams are then "attached" to slice $i(z)$ : exponential factors $M^{-(i(z)-l)}$ at least are obtained if $l<i(z)$, where $l$ is the highest internal line of the diagram. The only possible exceptions are diagrams in which the external line 1 and one of the external lines 3,4 arrive at the same vertex while 2 and the remaining line among 3,4 also arrive at a common vertex.

The result follows from the standard energy-momentum conservation arguments if 1 , or 2 , arrives at a vertex involving no other external line. On the other hand, leaving aside the trivial diagram with only one vertex involving $1, \ldots, 4$, 2-particle irreducibility excludes cases in which 1 and 2 would arrive at the same vertex (together possibly with 3 or 4 . We recall that contributing diagrams are also 1-particle irreducible). 
Remark 2. The above exceptional diagrams are independent of $k=p_{1}+p_{2}$ (in energy-momentum space).

Remark 3. In cases when $z^{\prime}$ is not close to $z$, e.g. $z$ large, $z^{\prime}$ fixed or $\left|z^{\prime}\right|<|z| / 3$, exceptional diagrams are removed by energy-momentum conservation: the energymomentum $p_{1}+p_{3}$ or $p_{1}+p_{4}$ is equal to $z \pm z^{\prime}$. If $\left|z^{\prime}\right|<|z| / 3$, then $\left|z \pm z^{\prime}\right|$ remains of the order of $|z|$.

We now give the proof of the first part of Proposition 1 (Eqs. (2) (2')). For completeness, we also prove the further bound:

$$
\left|G\left(k, z, z^{\prime}\right)-\lambda_{i(z)}\right|<\operatorname{Inf}\left\{\lambda_{\text {ren }}^{2}, \frac{1}{\ln (1+|z|)^{2}}\right\} \quad \text { if } \quad\left|z^{\prime}\right| \leqq|z| / 3 .
$$

Proof of the Bounds (2), (2'),(32). We start with (2). The effective coupling of a vertex involving 1 or 2 is $\leqq \lambda_{i(z)}$, which behaves like $1 / \ln |z|$ at large $z$, as follows from the analysis of [IM2] complemented by Remarks 1,3. It is also $\leqq \lambda_{\text {ren }}$. Equation (2) follows. If the trivial diagram with one vertex (whose value is $\lambda_{i(z)}$ ) is removed, diagrams involved have at least two vertices involving 1 and 2 separately. Equation (32) follows. Finally $\left(2^{\prime}\right)$ is the same bound as that obtained on $F$. It might be improved (in a limited way) since the channel $1,2 \rightarrow 3,4$ is not relevant here in the analysis, but channels $1,3 \rightarrow 2,4$ and $1,4 \rightarrow 2,3$ are (energy-momentum conservation does not apply in general, and 2-particle irreducibility in the channel 1,2 $\rightarrow 3,4$ is not useful in these cases).

Remark. The bound (2) and the result on $F$ of [IM2] allow one to check directly that the integral $G \circ F$ in the BS equation $\left(G \circ F\left(k, z, z^{\prime}\right)=\int G(k, z, \zeta) F\left(k, \zeta, z^{\prime}\right)\right.$ $\omega(k, \zeta) d \zeta)$ is convergent. Convergence follows from the decrease in $1 / \ln |\zeta|$ and $1 / \ln |\zeta|^{1-\sigma}$ of $G$ and $F$ respectively as $\zeta \rightarrow \infty$ (while $\omega(k, \zeta)$ decreases like $1 /|\zeta|^{2}$ ).

We next establish Part 2) of Proposition 1.

Proof of (3), $\left(3^{\prime}\right),\left(3^{\prime \prime}\right)$.

a) Proof of (3). Taking the difference $G-G^{\circ}$ has the following effects:

(i) It ensures in view of Remark 2 that all contributing diagrams are now "attached" to slice $i(z)$, hence factors in $M^{-(i(z)-j)}$ are always obtained if $j$ is the lowest line of the diagram (taking into account the factors $M^{-(l-j)}$.

(ii) At the cost of possible powers of $|k|$ (arising from the action of gradients), it ensures further factors $M^{-j}$ in the usual way applied in the renormalization procedures.

The combination of effects (i) and (ii) provides factors in $M^{-i(z)}$ for all diagrams. If a part in $M^{-\varepsilon i(z)}$ is kept for internal resummations, a decrease in $M^{-(1-\varepsilon) i(z)}$ is left. Equation (3) follows. (A factor $\lambda_{\text {ren }}^{2}$ can always be obtained since there are at least two couplings in all diagrams. On the other hand, the restriction to $\lambda_{\text {ren }}<\lambda_{0} \varepsilon$ is due to the fact that $\sum_{i} M^{-\varepsilon i} \approx \mathrm{cst} / \varepsilon$. For each internal resummation, a factor $1 / \varepsilon$ is thus obtained. It can be compensated by the factor $\varepsilon$ in the bound on one of the couplings, convergence properties being thus preserved at small $\lambda_{0}$.)

b) Proof of ( $\left.3^{\prime}\right)$. We establish below ( $\left.3^{\prime}\right)$ for $\left|z^{\prime}\right| \leqq|z| / 3$ (otherwise, the result follows from bounds on $|G|$ and $\left|G_{0}\right|$ separately). Under this condition, there are no exceptional diagrams as already remarked, so that factors $M^{-(i(z)-j)}$ are obtained 
for all diagrams. Taking the difference $G-G_{0}$ now yields factors in $M^{-\left(j-i\left(z^{\prime}\right)\right)}$ if $i\left(z^{\prime}\right)<j$. The combination gives a factor $M^{-\left(i(z)-i\left(z^{\prime}\right)\right)}$. A part $M^{-\varepsilon\left(i(z)-i\left(z^{\prime}\right)\right)}$ is kept as in a) for internal resummations.

c) Proof of $\left(3^{\prime \prime}\right)$. Equation ( $\left.3^{\prime \prime}\right)$ follows from a direct adaptation of previous methods. A factor $M^{-i(z)}$ is obtained for all diagrams, without subtraction, since there are at least six outgoing lines (counting external lines) for cuttings below the lowest internal slice of contributing diagrams (external lines $3, \ldots, N+2$ can be considered by convention to belong to slice 1 since the behaviour with respect to $z^{\prime}$ is not studied here).

\section{Remarks}

1. The combination of (3) and (3') gives for $\left|z^{\prime}\right| \leqq|z|$

$$
G\left(k, z, z^{\prime}\right)=G(0, z, 0)+R_{G}\left(k, z, z^{\prime}\right),
$$

where $R_{G} \equiv G-\dot{G}_{0}$ satisfies the bound

$$
\left|R_{G}\left(k, z, z^{\prime}\right)\right|<f^{\prime}(k) \lambda_{\text {ren }}^{2}\left(\frac{1+\left|z^{\prime}\right|}{1+|z|}\right)^{1-\varepsilon}
$$

i.e. decreases at least like $1 /|z|^{1-\varepsilon}$ as $z \rightarrow \infty$.

This result is a simplified version for $G$ of the Wilson short-distance expansion (at first order and "up to $\varepsilon$ ") to be established in turn for $F\left(k, z, z^{\prime}\right)$. It can also be established along the lines used in [IM2] for $F$, the analysis being now trivialized: the factorization $F\left(k, z, z^{\prime}\right)=\sum_{j=1}^{i(z)} \lambda_{j} F^{(j)}\left(k, z^{\prime}\right)+F^{\prime}\left(k, z, z^{\prime}\right)$ of [IM2] reduces in fact to

$$
G\left(k, z, z^{\prime}\right)=\lambda_{1}(z)+R_{G}^{\prime}\left(k, z, z^{\prime}\right)
$$

where $R^{\prime}$ decreases at least like $1 /|z|^{1-\varepsilon}$. On the other hand, restricting (35) to $k=z^{\prime}=0$ gives

$$
\lambda_{1}(z)=G(0, z, 0)-R_{G}^{\prime}(0, z, 0),
$$

from which (33) follows with $R_{G}=R_{G}^{\prime}\left(k, z, z^{\prime}\right)-R_{G}^{\prime}(0, z, 0)$.

2. The bounds (2), $\left(2^{\prime}\right),(3)$ hold similarly with $z$ replaced by $z^{\prime}$ if $\left|z^{\prime}\right| \geqq|z|$. In the case of $G-G_{0}$, the situation is different (since $z, z^{\prime}$ do not play a similar role in this difference). At $\left|z^{\prime}\right| \geqq|z|$, we shall use later the bounds provided by Proposition 1 on $G$ and $G_{0}$ separately.

\section{Applications}

5.1. Symanzik's Equations and Wilson Short-Distance Expansion. We first state the following lemma, which will be useful below to define $\dot{R}_{4}$ from its expression (15) in terms of $B$ and to obtain its decrease properties.

Lemma 3. Let $\stackrel{B}{B}\left(z, z^{\prime}\right), \stackrel{\oplus}{(\zeta)}$ satisfy for some $\varepsilon>0$ the bounds:

$$
\left|\stackrel{B}{B}\left(z, z^{\prime}\right)\right|<\operatorname{cst} \lambda_{\text {ren }}^{2}\left(\frac{1+\left|z^{\prime}\right|}{1+|z|}\right)^{1-\varepsilon}, \quad\left|z^{\prime}\right| \leqq|z|,
$$




$$
\begin{aligned}
& \left|\stackrel{\circ}{B}\left(z, z^{\prime}\right)\right|<\operatorname{cst} \lambda_{\text {ren }}, \quad z^{\prime} \geqq|z|, \\
& |ळ(\zeta)|<\frac{\text { cst }}{1+|\zeta|^{2}},
\end{aligned}
$$

for $\lambda_{\text {ren }}<\lambda_{0} \varepsilon$. Then the series (15) is absolutely convergent for $\lambda_{\text {ren }}<\lambda_{0}^{\prime} \varepsilon\left(\lambda_{0}^{\prime}>0\right)$ and

$$
\left|\AA^{\circ}\left(z, z^{\prime}\right)\right|<\operatorname{cst} \lambda_{\text {ren }}^{2}\left(\frac{1+\left|z^{\prime}\right|}{1+|z|}\right)^{1-2 \varepsilon}
$$

for $\left|z^{\prime}\right| \leqq|z|$.

\section{Remarks}

1. Lemma 3 indicates in particular that $\stackrel{R}{4}_{4}$ decreases at least like $1 /|z|^{1-2 \varepsilon}$ as $z \rightarrow \infty, z^{\prime}$ fixed. For later purposes, we note that the following bound follows also from the assumptions of Lemma 3 at $\left|z^{\prime}\right| \geqq|z|$ :

$$
\left|R_{4}^{\circ}\left(z, z^{\prime}\right)\right|<\lambda_{\text {ren }}\left(\frac{1+\left|z^{\prime}\right|}{1+|z|}\right)^{2 \varepsilon} \text {. }
$$

2. The bound (42) on $\stackrel{\circ}{B}$ is satisfied in view of Proposition 1 , Eqs. (3), $\left(3^{\prime}\right)$. The bound (43) follow from the bounds on $\stackrel{\circ}{G}$ and $\stackrel{\circ}{G}_{0}$ separately provided by Proposition 1, Eqs. (2) (2'). Equation (44) follows from the results of [IM1] on the 2-point function. These results can also be established similarly in the framework of this paper ("particle analysis" in all momentum slices).

3. The replacement of the bound (43) by

$$
\left|\stackrel{B}{B}\left(z, z^{\prime}\right)\right| \leqq \operatorname{cst} \lambda_{\text {ren }}\left(\frac{1+\left|z^{\prime}\right|}{1+|z|}\right)^{\varepsilon}, \quad\left|z^{\prime}\right| \geqq z
$$

would only change $\lambda_{0}^{\prime}$.

This formulation might allow one to cover hypothetical à la $\varphi_{4}^{4}$ theories in which asymptotic freedom would not be satisfied (with $G$ possibly replaced by $G_{\text {ren }}$ : see note at the end of Sect. 1.2).

Proof of Lemma 3 (and of (46)). The following bounds are established by induction on $n$, on the terms $\dot{B}^{(n)}=\stackrel{B}{ } \leftrightarrow \cdots \cdots \stackrel{\circ}{\omega}(n$ factors $B)$ :

$$
\begin{array}{ll}
\left|\dot{B}^{(n)}\left(z, z^{\prime}\right)\right|<C_{1}\left(C_{2} \lambda_{\text {ren }}\right)^{n}\left(\frac{1+\left|z^{\prime}\right|}{1+|z|}\right)^{1-2 \varepsilon} \cdot & \left|z^{\prime}\right| \leqq|z|, \\
\left|\dot{B}^{(n)}\left(z, z^{\prime}\right)\right|<C_{1}\left(C_{2} \lambda_{\text {ren }}\right)^{n}\left(\frac{1+\left|z^{\prime}\right|}{1+|z|}\right)^{2 \varepsilon}, & \left|z^{\prime}\right| \geqq|z|,
\end{array}
$$

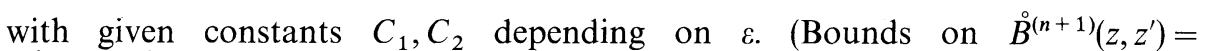
$\int \dot{B}^{(n)}(z, \zeta) \dot{B}\left(\zeta, z^{\prime}\right) \dot{\omega}(\zeta) d \zeta$ follow from those on $\dot{B}^{(n)}$ assumed by induction, and from the bounds (42) (43) (44), by a suitable division of the integration domain in $\zeta$ ).

The bounds (45) (46) follow by resummation over $n$.

We now give the proof of Proposition 1.1, first for euclidean values of $k, z^{\prime}$ (and $z$ ). See in Sect. 5.2 extensions to values of $k$ up to $s=16 \mu^{2}-\eta$ in Minkowski space. 
Proof of Proposition 1.1. Symanzik's equations can be established in a rigorous way as follows. We first start with a finite ultraviolet cut-off $\rho$ in the theory. The equations are then established at sufficiently small values of the bare coupling $\lambda$, e.g. in a region of the form $|\lambda|<\operatorname{cst} M^{(\rho+\varepsilon) / 2}$ : the formal proofs of Sect. 4 are rigorous, all integrals being convergent (in view of the cut-off) and all series being also convergent in view of the factors $|\lambda|$ included in the bounds on $|G|$.

From the known analyticity of $F_{N+2}^{(\rho)}, G^{(\rho)} ; L_{N+2}^{(\rho)}$ in $\lambda$, all terms and all equations can in turn be analytically continued in $\lambda$ up to the value (25) of $\lambda_{\rho}$. The existence and analytically in $\lambda$ of $R_{4}$ follow, by a slight adaptation of Lemma 3, from those of $B^{\circ}$; uniform bounds of the form given in Sect. 3 are in fact obtained for all finite values of $\rho$ on the terms $B^{(\rho)}\left(\zeta_{i}, \zeta_{i+1}\right)$ involved in convolution integrals for $\left|\zeta_{i}\right|$, $\left|\zeta_{i+1}\right|<M^{2 \rho}$, or bounds in cst $\left|\lambda_{\text {ren }}\right|$ otherwise. On the other hand, cut-off factors in $e^{-M^{-2 \rho} \zeta_{i}^{2}}$ are contained in $\omega^{(\rho)}\left(0, \zeta_{i}\right)$. Results follow in turn for $R_{4}$ and $R_{N+2}$ from (16), (17). Finally the $\rho \rightarrow \infty$ limits are obtained again from the uniform bounds

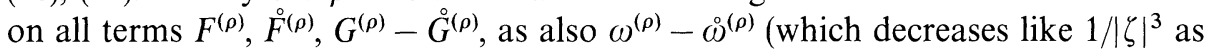
$\zeta \rightarrow \infty)$. The fact that the terms have well defined limits follows from usual arguments. The term $\Lambda_{N}$ is well defined in the $\rho \rightarrow \infty$ limit as a consequence of (18) and of the bounds on $R_{N+2}$.

Remark. If one starts from Proposition 2 as stated in Sect. 1, the proof of Proposition 2.1 is similar to above. We note that the convolution integral

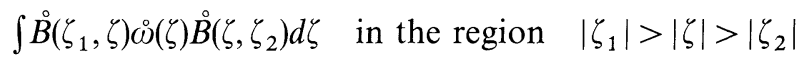

converges uniformly in $\zeta_{1}$ due to the factor $1 /\left(\ln \zeta_{1}\right)^{2}$ of bound $\left(5^{\prime}\right)$.

\subsection{2-Particle Asymptotic Completeness and Related Results (Outline)}

Analytic Continuation of the 4-Point Function. The momentum space 4-point function $F$ is initially defined and analytic in a strip around euclidean space going up to $s=4 \mu^{2}-\eta(\eta$ can be chosen arbitrarily small but maximal possible values of $\lambda_{\text {ren }}$ tend to zero as $\eta \rightarrow 0$ ). From its definition of Sect. 3 and its "4-particle exponential decay" in euclidean space-time (arising from 2-particle irreducibility with respect to propagators of the lowest momentum slice), $G$ is analytic in a larger strip going up to $s=16 \mu^{2}-\eta$ in Minkowski space.

In super-renormalizable theories like $P(\varphi)_{2}, F$ can in turn be analytically continued [B, BL, SZ, BI] as a meromorphic function (with possible poles in $s$ ) in a 2-sheeted domain around $s=4 \mu^{2}$, going up to $16 \mu^{2}-\eta$ in Minkowski space; 2-particle AC also follows in the region $s$ real, $4 \mu^{2}<s<16 \mu^{2}-\eta$.

In the GN model, the BS equation is still valid, as we have seen, in euclidean space. However, it does not allow one to obtain the desired analytic continuation of $F$. This is linked with the "weakness" of the convergence of the integral $G \circ F$ or correspondingly the fact that terms $G \circ \ldots \circ G$ ( $n$ factors $G$ ) grow faster than any exponential in $n$ as $n \rightarrow \infty$. One may then wish, as suggested e.g. in [11], to first consider the BS equation

$$
F^{(\rho)}=G^{(\rho)}+F^{(\rho)} \omega^{(\rho)} G^{(\rho)}
$$

in the theory with ultraviolet cut-off. In view of the cut-off factor in $\omega_{\rho}$, the methods of $[\mathrm{B}, \mathrm{BL}]$ (and the uniform bounds on $G^{(\rho)}$ and on 2-point functions that follow 
from the methods of this paper) allow one to make the analytic continuation of $F^{(\rho)}$. However, possible poles of $F^{(\rho)}$, which depend on $\rho$, cannot be controlled in the absence of further information: some of these poles might lie on the real axis or close to it and they might moreover accumulate here in the $\rho \rightarrow \infty$ limit.

To control the behaviour of $F^{(\rho)}$ or $F$, we then have recourse (following the suggestion of $[\mathrm{BD}]$ ) to Eq. (20) which can be rewritten (at $\rho$ finite or infinite), at first in a formal sense, as:

$$
\begin{aligned}
& F=[1-\stackrel{\circ}{F}(\omega-\stackrel{\circ}{\omega})-(1-\stackrel{\circ}{F} \circ)(G-\stackrel{\circ}{G}) \omega]^{-1}[\stackrel{\circ}{F}+(1+\stackrel{\circ}{F} \circ)(G-\stackrel{\circ}{G})] \\
& =[1+\stackrel{\circ}{F}(\omega-\stackrel{\circ}{\omega})+(1+\stackrel{\circ}{F} \stackrel{\circ}{)}(G-\stackrel{\circ}{G}) \omega+\cdots][\stackrel{\circ}{F}+(1+\stackrel{\circ}{F} \stackrel{\circ}{\circ})(G-\stackrel{\circ}{G})]
\end{aligned}
$$

(where $\rho$ is left implicit).

Using the analyticity of $G$, the bounds on $\stackrel{\circ}{F}, G-\stackrel{\circ}{G}, \omega-\stackrel{\circ}{\omega}$ and the same methods as in Sect. 5.1 completed in the standard way [B] by suitable local distortions of integration contours, Eq. (51) allows one to define $F^{(\rho)}$, at small coupling, or $F$ (at $\rho$ infinite) as a bounded, hence analytic function with no pole in a given (2-sheeted) neighborhood of the real axis independent of $\rho$, going up to $16 \mu^{2}-\eta$ in Minkowski space, away from a complex neighborhood of $s=4 \mu^{2}$ : individual integrals exist and relevant series are then convergent at small enough $\lambda_{\text {ren }}$.

A neighborhood of the 2-particle threshold is a priori excluded because, as well known, the 2-particle convolutions generate in dimension 2 kinematical factors $\left(4 \mu^{2}-s\right)^{-1 / 2}$ which yield divergences however small the coupling is. We below explain how this neighborhood can be treated by an adaptation of the methods of $[\mathrm{I} 2, \mathrm{BI}]$. We shall then explain how 2-particle asymptotic completeness is obtained.

The Neighborhood of the 2-Particle Threshold. For values of $s$ in a given complex neighborhood $V$ of $s=4 \mu^{2}$ (which can be chosen independent of the coupling), we introduce the same kernel $U$ as in [I2, BI] but in a different way. $U$ was defined in these works in terms of $\widehat{G}$ by the equation:

$$
U=\hat{G}+U \nabla_{M} \hat{G},
$$

where $\nabla_{M}=o_{M}-\frac{1}{2} *$ is regular at threshold in contrast to $o_{M}(*$ denotes on mass-shell-convolution). From (52), $U$ is shown to be analytic for $s$ in $V$, as the convergent sum (at small coupling) of the series $\widehat{G}+\widehat{G} \nabla_{M} \widehat{G}+\cdots$ and it satisfies:

$$
F=U+\frac{1}{2} U * F,
$$

which shows in particular that $U$ is independent of $M$ (in contrast to $\hat{G}$ ). In the present approach, where we wish to use the kernel $G$ but not $\hat{G}, U$ will still be shown to satisfy the equation:

$$
U=G+U \nabla G
$$

where $\nabla=o-\frac{1}{2} *$. But it cannot be directly defined (at $\rho$ infinite) from this equation: problems close to $s=4 \mu^{2}$ are not present in $\nabla$, but ultraviolet problems (as the intermediate variable $\zeta$ in the convolution $U \nabla G$ tends to infinity in euclidean space) are. $U$ is then defined as follows:

(i) For $s$ in $V$, but sufficiently below $4 \mu^{2}, U$ is defined as the convergent sum 
(at small coupling) of the series:

$$
U=F-\frac{1}{2} F * F+\cdots
$$

It can also be defined by an explicit formula derived from (53) and expressing $U$ in terms of $F$ : the latter is similar to that expressing $F$ in terms of $U$ in [BI].

(ii) Given $k_{0}$ such that $s_{0}=-k_{0}^{2}$ belongs to $V, U$ satisfies the relation analogous to (51) with $F$ replaced by $U$ and $\stackrel{\circ}{F}, \dot{G}$, replaced by the values of $U$ and $G$ at $k_{0} ; \varrho \equiv \omega(0, \zeta)$ is replaced by $\omega\left(k_{0}, \zeta\right)$ and integration contours $(\Gamma(k)$ and euclidean space respectively) are replaced by the invariant contour $\underline{\Gamma}$ (see [BI]), which is not pinched at threshold. (The operation $\nabla$ corresponds to integration over this contour.)

This relation (together with bounds on $G-G\left(k_{0}\right)$ analogous to those on $G-G^{\circ}$ in Sect. 4) now allows one to extend $U$ as an analytic function for $s$ in $V$. The expression of $F$ in terms of $U$ that follows from (53), initially valid for the values (i) of $s$ then allows one to make the analytic continuation of $F$ for $s$ in $V$ (in a 2-sheeted domain). The same results as in [BI] then follow. In particular $F$ has no pole at $s$ real, $4 \mu^{2}<s<16 \mu^{2}-\eta$.

2-Particle Asymptotic Completeness. We next show how 2-particle AC is then obtained. We first recall the algebraic argument of $[\mathrm{B}, \mathrm{BL}]$ in a somewhat more convenient from. It is a priori only formal in the present situation. We start from the BS equation assumed to hold at $s$ real, $s>4 \mu^{2}$, on the boundaries of the physical sheet from the sides $\operatorname{Im} s>0$ and $\operatorname{Im} s<0$ :

$$
\begin{aligned}
& F_{+}=G+F_{+} o_{+} G, \\
& F_{-}=G+G o_{-} F_{-} .
\end{aligned}
$$

Here, $F_{+}$and $F_{-}$are the corresponding boundary values of $F$ and $o_{+}, o_{-}$ correspond to integration contours $\Gamma_{+}, \Gamma_{-}$. From (56) (56'), one has:

$$
F_{+}-F_{-}=F_{+} * F_{-} .
$$

To obtain formally (57), replace $G$ in $(56)$ by $F_{-}-G o_{-} F_{-}$, use $F_{+} o_{+}\left(G o_{-} F_{-}\right)=$ $\left(F_{+} o_{+} G\right) o_{-} F_{-}=\left(F_{+}-G\right) o_{-} F_{-}$and the basic relation $[\mathrm{B}, \mathrm{BL}] o_{+}-o_{-}=*$.

Equation (57) does characterize 2-particle AC (see [B1, BL]).

Proof of (57). The proof of (57) can be made rigorous in several ways:

(a) either directly at $\rho$ infinite, using large momentum properties of $G(k, z, \zeta)$ (decrease in $1 / \ln |\zeta|$ as $\zeta \rightarrow \infty$ ) for values of $k$ up to $s=16 \mu^{2}-\eta$ and related properties of $F$ (decrease in $\left.1 /(\ln |\zeta|)^{1-\sigma}\right)$. The decrease of $G$ is established as in Sect. 3. The decrease of $F$ is obtained through Eq. (51) (using results on $\stackrel{\circ}{F}$ and $G-\stackrel{G}{G})$ away from the neighborhood of $s=4 \mu^{2}$, and from its expression in terms of $U$ for $s$ in $V$. (Properties of $U$ follow from its definition in terms of $G$.)

These decrease properties ensure that the BS equation still makes sense (by analytic continuation of all terms) in the domain of $F$ and that all operations involved in the algebraic argument are valid.

b) or by first working at finite values of $\rho$, starting from (50). The methods of 
[B, BL] yield the relation $\left(F^{(\rho)}\right)_{+}-\left(F^{(\rho)}\right)_{-}=\left(F^{(\rho)}\right)_{+} *\left(F^{(\rho)}\right)_{-}$outside possible poles of $F^{(\rho)}$. The use of Eq. (51) at any given $\rho$, the uniformity of the bounds on $G^{(\rho)}-G_{0}^{(\rho)}$ and the introduction of $U^{(\rho)}$ for $s$ in $V$, allow one to exclude poles of $F^{(\rho)}$ in a uniform domain (containing the region $4 \mu^{2}<s<16 \mu^{2}-\eta$ ) and hence to obtain the needed relation $F_{+}-F_{-}=F_{+} * F_{-}$in the $\rho \rightarrow \infty$ limit.

c) An alternative proposal has been suggested in [BD]: work at $\rho$ infinite and derive the relation $F_{+}-F_{-}=F_{+} * F_{-}$directly from (51), without using the BS equation. An algebraic argument is given to that purpose in [BD]. This approach can also be made rigorous in a way similar to above. However, it is somewhat more complicated and is thus omitted.

Methods b), c) would in principle apply also to theories in which the BS kernel would not exist in the $\rho \rightarrow \infty$ limit, but in which one would have suitable properties of $G_{\mathrm{ren}}^{(\rho)}-\dot{G}_{\mathrm{ren}}^{(\rho)}$ at $\rho$ finite or infinite.

Wilson Short-Distance Expansion (Minkowskian Values of $k$ ). We conclude this section with the extension of the Wilson short-distance expansion at first order to values of $k$ up to $s=16 \mu^{2}-\eta$ in Minkowski space. The extension is based on the properties of $G, G-G$ and on formula (51), at least outside the neighborhood of the 2-particle threshold. The latter is treated via the introducing of the kernel $U$ (and the analysis of its properties from those of $G$ ).

Results are first established for $F_{4}$, hence for $R_{4}$. They hold in turn for $F_{N+2}$ via Eq. (17) from results on $L_{N+2}$ (see Sect. 4).

Remark. The term $R_{4} \omega L_{N+2}$ can be seen to be bounded as $s \rightarrow 4 \mu^{2}$, using $o=\nabla+\frac{1}{2} *$ and noting that the restriction $R_{4}$ of $R_{4}$ to the mass shell on the right contains a factor $\sigma^{+1 / 2}$ as $\sigma=4 \mu^{2}-s \rightarrow 0$. This factor compensates the factor $\sigma^{-1 / 2}$ generated by $*$. This property of $R_{4 \mid}$ follows from the expression of $F_{4 \mid}$ in terms of $U$ (see [BI]) and of the Wilson type expansion of $U$ derived from its definition in terms of $G$, which gives in turn an adequate expression of $R_{4}$.

\section{Discussion}

It has been emphasized in [BD] that the use of the "renormalized" BS kernel $G_{\text {ren }}$ might allow one, for "à la $\varphi_{4}^{4}$ " theories, to treat both Wilson short distance expression at first order ( $=$ factorization properties at large momentum in euclidean space) and 2-particle asymptotic completeness and related results (= local properties in complex energy-momentum space including the 2-particle region in Minkowski space).

We have seen in this work that this is indeed the case for the weakly coupled massive Gross-Neveu model (where one may use either the true BS kernel $G$ or $\left.G_{\text {ren }}\right)$, in view of different properties of the 2-p.i. phase-space diagrams contributing to $G$. We now compare the methods of [IM1,IM2] with those of this paper on the two topics.

Asymptotic Completeness and Related Results. The present method is more complicated than that of [IM1] and in fact obliges one to treat ultraviolet problems that are not really relevant to the local problems (in complex momentum space) 
under study. However, this approach may have its own interest, in particular if one is interested in extensions to higher energies as we now discuss. In this case, " $n$-particle" irreducible kernels have to be introduced. The method of [IM1], ("particle analysis" made only in slice 1) still applies because propagators of slices $>1$, which have no pole singularity, fall off faster than any exponential in euclidean space-time. Thus, they satisfy in particular bounds in $C_{n} e^{-n \mu|x|}, \forall n$, and diagrams that are 2-p.i. with respect to propagators of slice 1 but can be cut into 2 parts by cutting, e.g. one or two propagator lines in slices $>1$ do not prevent " $n$-particle exponential decay" or the analyticity properties in complex energy-momentum space that follow by the Fourier-Laplace transformation.

For any given energy region one wishes to explore in Minkowski space, there is a maximal relevant value of $n$ and the dependence of $C_{n}$ on $n$ can be compensated by taking the coupling $\lambda_{\text {ren }}$ sufficiently small. However, it will have to be smaller and smaller as the energy increases. To remove this problem, "particle analysis" in slices $>1$ seems needed. The use of "particle analysis" in all slices from the outset, as done in this paper for the BS kernel $G$, might provide an elegant solution.

We recall, however, that there are also, so far, other unsolved difficulties in the program. (See more details in [IM2, 3, I3].)

Wilson Short-Distance Expansion. We first consider Wilson expansion at first order and "up to $\varepsilon$ " (decrease in $1 /|z|^{1-\varepsilon}$ of $R_{N+2}$ for $\lambda_{\text {ren }}<\lambda_{0} \varepsilon$ ) and restrict our discussion to euclidean values of all variables. The method of [IM2] and that developed in this paper are two different but related ways of extracting desired results by suitable expansions of $R_{N+2}$ in terms of "irreducible" kernels. Relevant irreducibility in [IM2] is with respect to different slices only, and corresponding expansions involve summations over slice indices of these kernels. The BS kernel used in this paper is a more intrinsic quantity and expansions are obtained in terms of actual Feynman type convolutions with no reference to the details of phase space analysis. Besides, the use of $G$ allows one to treat in essentially the same way euclidean values of $k$, or Minkowskian values of $k$ in the 2-particle region.

We note, however, that the methods of [IM2] are more direct from a constructive viewpoint, are valid in principle for more general theories and allow expansions to higher orders, while the method based on the BS kernel $G$ is relevant only for à la $\varphi_{4}^{4}$ theories (in view of the connection between 2-particle irreducibility and the fact that renormalization parts are at most 4-point functions) and at first order. These restrictions might be overcome by introducing higher order irreducible kernels, but these kernels would be anyway more complicated than those involved in higher particle expansions.

As a general conclusion, the fact that the same kernel and equation serves two purposes, namely proving Wilson expansion on the one hand, asymptotic completeness and related results on the other hand is limited to the first order and to the 2-particle region respectively. 


\section{References}

[B] Bros, J : Analytic methods on mathematical physics. Newton, G. (ed.). New-York. Gordon and Breach 1970, p. 85

[BD] Bros, J., Ducomet, B.: Ann. Inst. Henri Poincaré 45, 2, 173 (1986)

[BI] Bros, J., Iagolnitzer, D.: 2-particle asymptotic completeness and bound states in weakly coupled quantum field theories. Commun. Math. Phys. (in press)

[BL] Bros, J., Lassalle, M.: Analyticity properties and many-particle structure in general quantum field theory. III. Two-particle irreducibility in a single channel. Commun. Math. Phys. 54, 33 (1977)

[FMRS] Feldman, J., Magnen, J., Rivasseau, V., Sénéor, R., A renormalizable field theory: The massive Gross-Neveu model in two dimensions. Commun. Math. Phys. 103, 67 (1986)

[GK] Gawedzki, K., Kupiainen, A.: Massless lattice $\varphi_{4}^{4}$ theory: Rigorous control of a renormalizable asymptotically free model. Commun. Math. Phys. 99, 197 (1985)

[GN] Gross, D., Neveu, A.: Phys. Rev. D10, 3235 (1974)

[I1] Iagolnitzer, D.: Regularized and renormalized Bethe-Salpeter equations: Some aspects of irreducibility and asymptotic completeness in renormalizable theories. Commun. Math. Phys. 99, 451 (1985), Fizika 17, 3 (1985)

[I2] Iagolnitzer, D.: Irreducible kernels and nonperturbative expansions in a theory with pure $m \rightarrow m$ interaction.: Commun. Math. Phys. 88, 235 (1983)

[13] Iagolnitzer, D.: VIIh. International Congress of Mathematical Physics. Mebkhout, M. Sénéor, R. (eds.) Singapore: World Scientific 1987, p. 622

[IM1] Iagolnitzer, D., Magnen, J.: Asymptotic completeness and multiparticle structure in field theories. II. Theories with renormalization: The Gross-Neveu model. Commun. Math. Phys. 111, 81 (1987)

[IM2] Iagolnitzer, D., Magnen, J.: Large momentum properties and Wilson short-distance expansion in non-perturbative field theory. Commun. Math. Phys. 119, 609-626 (1988)

[IM3] Iagolnitzer, D., Magnen, J.: Asymptotic completeness and multiparticle structure in field theories. Commun. Math. Phys. 110, 51 (1987)

[MW] Mitter, P., Weisz, P.M.: Phys. Rev. D8, 4410 (1973)

[SZ] Spencer, T., Zirilli, F.: Scattering states and bound states in $\lambda P(\phi)_{2}$. Commun. Math. Phys. 49, 1 (1976)

[S] Symanzik, K.: Small-distance-behaviour analysis and Wilson expansions, Commun. Math. Phys. 23, 49 (1971), Infrared singularities and small-distance-behaviour analysis. 34, 7 (1973)

[W] Wilson, K.: Phys. Rev. 179, 1499 (1969)

[Z] Zimmerman, W.: Ann. Phys. 77, 536 (1973)

Communicated by K. Gawedzki

Received May 4, 1988 